\title{
A Closer Look at the Bivariate Association between Ambient Air Pollution and Allergic Diseases: The Role of Spatial Analysis
}

\author{
Dohyeong Kim ${ }^{1}$ (D), SungChul Seo ${ }^{2, *}$ (1) , Soojin Min ${ }^{1}$, Zachary Simoni ${ }^{1}$, Seunghyun Kim ${ }^{3}$ \\ and Myoungkon Kim ${ }^{3}$ \\ 1 School of Economic, Political and Policy Sciences, The University of Texas at Dallas, 800 W Campbell Road, \\ Richardson, TX 75080, USA; dohyeong.kim@utdallas.edu (D.K.); soojin.min@utdallas.edu (S.M.); \\ zrs160030@utdallas.edu (Z.S.) \\ 2 Department of Environmental Health and Safety, College of Health Industry, Eulji University, 553 \\ Sanseong-Daero, Sujeong-Gu, Seongnam-Si, Gyeonggi-Do 13135, Korea \\ 3 Department of Medical Biochemistry \& Molecular Biology, Korea University, Anam-dong, Seongbuk-gu, \\ Seoul 136-701, Korea; seunghyun@korea.ac.kr (S.K.); jerrykim@korea.ac.kr (M.K.) \\ * Correspondence: sseo@cup.ac.kr; Tel.: +82-31-740-7230
}

Received: 26 June 2018; Accepted: 30 July 2018; Published: 1 August 2018

\begin{abstract}
Although previous ecological studies investigating the association between air pollution and allergic diseases accounted for temporal or seasonal relationships, few studies address spatial non-stationarity or autocorrelation explicitly. Our objective was to examine bivariate correlation between outdoor air pollutants and the prevalence of allergic diseases, highlighting the limitation of a non-spatial correlation measure, and suggesting an alternative to address spatial autocorrelation. The 5-year prevalence data (2011-2015) of allergic rhinitis, atopic dermatitis, and asthma were integrated with the measures of four major air pollutants $\left(\mathrm{SO}_{2}, \mathrm{NO}_{2}, \mathrm{CO}\right.$, and $\left.\mathrm{PM}_{10}\right)$ for each of the 423 sub-districts of Seoul. Lee's L statistics, which captures how much bivariate associations are spatially clustered, was calculated and compared with Pearson's correlation coefficient for each pair of the air pollutants and allergic diseases. A series of maps showing spatiotemporal patterns of allergic diseases at the sub-district level reveals a substantial degree of spatial heterogeneity. A high spatial autocorrelation was observed for all pollutants and diseases, leading to significant dissimilarities between the two bivariate association measures. The local $L$ statistics identifies the areas where a specific air pollutant is considered to be contributing to a type of allergic disease. This study suggests that a bivariate correlation measure between air pollutants and allergic diseases should capture spatially-clustered phenomenon of the association, and detect the local instability in their relationships. It highlights the role of spatial analysis in investigating the contribution of the local-level spatiotemporal dynamics of air pollution to trends and the distribution of allergic diseases.
\end{abstract}

Keywords: allergic disease; air pollution; bivariate association; Geographic Information Systems; spatial analysis

\section{Introduction}

Although numerous studies report that genetic factors play a critical role in determining susceptibility or exacerbation of allergic diseases such as atopic dermatitis, asthma, and allergic rhinitis [1], recent work has been dedicated to environmental exposure to better understand the prevalence of allergic diseases, since a significant change in human genetics could not occur in such a short period [2]. The increased prevalence of allergic diseases has been found in "westernized" or "modernized" countries such as South Korea, and this epidemiologic change has been attributed to the 
augmented level of air pollution in those countries [3]. Most of the research investigates ambient air pollutants, including particle matters $\left(\mathrm{PM}_{10}\right.$ and $\left.\mathrm{PM}_{2.5}\right)$, nitrogen dioxide $\left(\mathrm{NO}_{2}\right)$, carbon monoxide (CO), sulfur dioxide $\left(\mathrm{SO}_{2}\right)$ and ozone $\left(\mathrm{O}_{3}\right)$ [4]. Although the majority of studies investigating the relationship between air pollutants and allergic diseases have been cross-sectional, observational, or longitudinal cohort studies, there are relatively few ecological studies [5] due to susceptibility to the ecological fallacy. The ecological fallacy refers to the possibility of making misleading inferences based on grouped individuals [6]. Moreover, as areal data is often used in spatial analysis, the ecological fallacy may lead to the Modifiable Areal Unit Problem (MAUP), which produces widely varied analytical results depending on the level of spatial aggregation of data [7]. The MAUP effect causes the inference to change depending on the size of aggregated geographical areas or the rules of forming areal units [8]. One study found that regional and local variations in asthma prevalence were not observed by national level data, since aggregation of data masks significant spatial variations in the outcomes [9]. Thus, using fine geographic granularity is recommended to adequately address the MAUP effect [10].

Although the MAUP effect is essentially a matter for all quantitative studies involving areal data [11], ecological research can be beneficial in a number of ways. First, readily available, timely data, often used by ecological research, allows low resource-intensive research. Due to this advantage, the ecological approach has been widely used in various environmental health studies [12-15]. It is also the case in research on air pollution related allergic diseases. Hospitals generally maintain electronic health records such as numbers of patient visits or admissions, whereas governments manage and release air pollution statistics on a regular basis. Some studies have used routinely-collected health records to explore the ecological connection between air pollution and allergic diseases which may have been neglected by previous individual-level studies [16,17]. Moreover, the ecological approach helps understand the intricate health effects of outdoor air pollution by comparing health outcomes in populations across gradient levels of air pollutants [18].

Although previous ecological studies investigating the association between air pollution and allergic diseases accounted for temporal or seasonal relationships [16,19-21], few studies consider spatial aspects in their analyses, including spatial autocorrelations. Ecological variables in geographically referenced data sets tend to be dependent from one another within a relatively close range due to spatial autocorrelation [22]. The information from spatially auto-correlated observations is different from independent observations, as a certain amount of the information from each observation is duplicated within the cluster [23]. Thus, because the presence of spatial autocorrelation is often observed in environment or health related variables [24,25], it must be addressed when studying the ecological association between air pollution and allergic diseases. Failure to do so will increase the likelihood of inaccurate statistical decisions such as Type I errors [22]. Therefore, addressing the nature of spatial heterogeneity of both environmental and health variables helps interpret the "true" effect between air pollutants and prevalence of allergic diseases. Due to these factors, spatial perspectives are vital for an adequate interpretation of ecological analyses using aggregated areal data. Research on air pollution-related allergic diseases needs to acknowledge such properties intrinsic to spatial data representing geographical variations of the prevalence of allergic diseases and levels of outdoor air pollutants. While only a few studies consider univariate or bivariate spatial autocorrelation when estimating the impact of air pollution on allergic diseases [26,27], no study has accounted for a combinational effect of spatial autocorrelation of each measure and exposure-to-disease correlation between air pollutants and the disease prevalence.

In South Korea, spatial analysis of allergic disease related research involving ambient air pollution is lacking. Only one recent study considered spatial non-stationarity in researching the association between allergic diseases and air pollutants, but ignored spatial autocorrelation [17]. Recent studies based on national level data reported that the prevalence of asthma and atopic dermatitis demonstrated decreasing prevalence in South Korea [28,29], but these findings could be due to the MAUP effect, where spatially varied prevalence at the finer levels of geography may have been masked by national 
level observation. There is no indication that the levels of ambient air pollutants have been substantially decreasing in South Korea [30], so unless a significant and steady decrease in the associated risk factors were present, this trend may be a temporary phenomenon. Although levels of air pollutants including $\mathrm{SO}_{2}, \mathrm{NO}_{2}$, and $\mathrm{PM}_{10}$ in major cities have not considerably increased in the last 10 years [31], air pollution in Seoul, particularly $\mathrm{PM}_{10}$, has increased more compared to other large cities such as Paris, Los Angeles, and London [32,33]. A small number of studies on allergic diseases conducted in Korea have taken into account spatial autocorrelation in ambient air pollutants [34,35], while others focus upon the prevalence of allergic diseases [36]. However, no studies simultaneously account for spatial autocorrelation in their bivariate analysis.

Thus, this study examines 5-year prevalence trends (2011-2015) of three allergic diseases-allergic rhinitis, atopic dermatitis, and asthma-in terms of regional differences, highlights several methodical limitations in the literature, and suggests an alternative to address them. It first investigates spatial autocorrelation in four major air pollutants $\left(\mathrm{SO}_{2}, \mathrm{NO}_{2}, \mathrm{CO}\right.$ and $\left.\mathrm{PM}_{10}\right)$ and the prevalence of allergic diseases among 423 sub-districts in Seoul. Next, bivariate correlation between each air pollutant and allergic disease prevalence is examined by Pearson's correlation coefficients and its alternative measure, which are compared to each other in a cross-sectional manner and its trend over time. Lastly, this study assesses the role of spatial analysis, and provides policy implications in terms of how to design public health policies in order to efficiently address the gravity of the prevalence of allergic diseases in South Korea.

\section{Materials and Methods}

Emergency room visits or admission counts have been used as an outcome measure of disease exacerbation, but mostly for severe or acute asthma, rather than for atopic dermatitis or allergic rhinitis [37,38]. In other words, those metrics are permissible for assessing the risk of short-term exposures to air pollutants, but are limited at explaining the long-term effects [39]. Allergic diseases are related to immune disorders attributed to genetic and environmental factors [40]; children with immature immune systems would be relatively more vulnerable to allergic diseases [41]. Thus, we used patient-count data only for children under 12 in order to investigate the long-term effect of air pollution exposure in early childhood. By using data collected by the National Health Insurance Corporation (NHIC) of South Korea from 2011 to 2015, we calculated the number of patients per 10,000 children under 12 for allergic rhinitis, atopic dermatitis, and asthma for each of the 423 sub-districts of Seoul. We chose sub-district as the unit of analysis because it is the most disaggregated administrative unit at which the patient count data is currently available in Seoul. A sub-district is the smallest level of urban government to have its own office and administrative staff in South Korea, and hence, many administrative and public data are collected at this bureaucratic level.

The classification of three types of allergic diseases was based on the disease codes defined by the 11th revision of the International Classification of Diseases (ICD-11): J30 for vasomotor and allergic rhinitis, L20 for atopic dermatitis, and J45-46 for asthma and status asthmaticus. Each patient was not counted more than once, even if the patient had received multiple treatments under the same disease code. We created a series of maps at the sub-district level for each year to examine whether spatial and temporal variations in the prevalence of each allergic disease were observable across the units. We found no significant difference in these data between male and female, so they were analyzed together in all subsequent analyses.

The ambient air pollution data collected by the Ministry of the Environment of Korea included daily measurements of four major air pollutants- $\mathrm{SO}_{2}(\mathrm{ppb}), \mathrm{NO}_{2}(\mathrm{ppb}), \mathrm{CO}(\mathrm{ppb})$, and $\mathrm{PM}_{10}$ $\left(\mu \mathrm{g} / \mathrm{m}^{3}\right.$ ) -at 25 monitoring stations in Seoul (Figure 1). $\mathrm{SO}_{2}, \mathrm{NO}_{2}$, and $\mathrm{CO}$ are measured every five minutes, and $\mathrm{PM}_{10}$ is monitored each hour. Then, the 1-h mean concentrations of all pollutants are recorded and uploaded to the data archive system. The sampling methods of each pollutant are as follows: $\mathrm{SO}_{2}$ the pulse U.V. fluorescence method; $\mathrm{NO}_{2}$ the chemilum inescent method; $\mathrm{CO}$ the non-dispersive infrared method; $\mathrm{PM}_{10}$ for $\beta$-ray absorption method. We examined how 
the concentration levels of each pollutant in Seoul have changed over time for the study period (2011-2015). As there is only one monitoring station for air pollution in each district of Seoul, it is very unrealistic and misleading to assume that the measurement at the monitoring station is the same within the district [42]. So, we estimated the annual average concentration level of each air pollutant for each year and for each sub-district to match the spatial scale of the patient count data using the ordinary kriging method, which has been widely used as a spatial interpolation tool for air pollution measures in the literature $[17,43]$. We applied the spherical model to the log-transformed data for interpolation, as its assumption tends to be consistent with the spatial distribution pattern of ambient air pollutants [44].

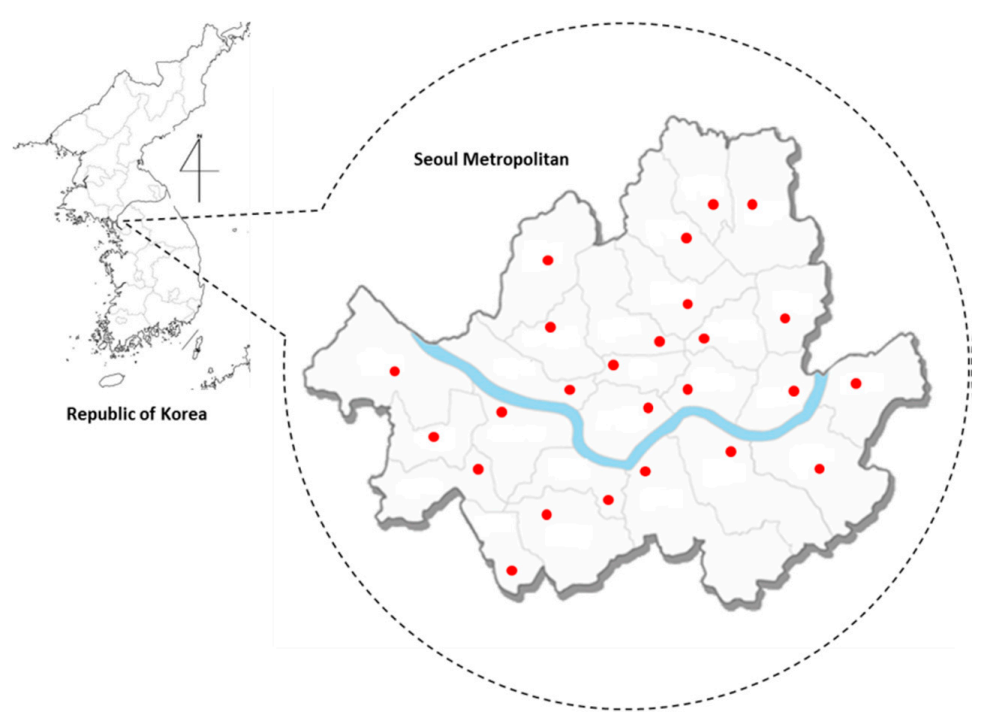

Figure 1. The distribution map of 25 monitoring stations for air pollutants (๑), one in each administrative district of Seoul.

We first checked if spatial heterogeneity in our main variables were consistent over the five years, and found the spatial patterns remained consistent over time. We were thus able to look into spatial and temporal heterogeneity separately. A univariate spatial autocorrelation was estimated for each allergic disease prevalence for each year and the corresponding air pollutant using a global Moran's coefficient, in order to evaluate whether or not there is a considerable degree of spatial autocorrelation in both data. With the interpolated measurements of the four pollutants in each sub-district, a series of bivariate correlation analyses were performed to scrutinize ecological associations between each ambient air pollutant and the prevalence of allergic diseases at the sub-district level in Seoul. Furthermore, we investigated how the associations changed over time. One of the classical methods of measuring a bivariate association is the Pearson's correlation coefficient, which can be written for variables $X$ and $Y$ as follows:

$$
r_{X, Y}=\frac{\sum_{i}\left(x_{i}-\bar{x}\right)\left(y_{i}-\bar{y}\right)}{\sqrt{\sum_{i}\left(x_{i}-\bar{x}\right)^{2}} \cdot \sqrt{\sum_{i}\left(y_{i}-\bar{y}\right)^{2}}}
$$

However, aspatial association measures, including Pearson's correlation coefficient, do not recognize the spatial phenomenon of data sets [23]. Thus, these measures face a serious shortcoming for hypothesis testing and prediction when bivariate associations are spatially clustered [45]. Although the bivariate spatial autocorrelation measures based on Wartenberg's original framework of multivariate spatial correlation (i.e., bivariate global and local Moran's I) have been widely used to examine the exposure-health relationship in various environmental health studies $[26,46,47]$ via the GeoDa 
package [48], they fail to incorporate spatial smoothing scalers of both $X$ and $Y$ variables. Rather, they can only gauge the relationship between a variable and the other variable's spatial lag [49].

As a better alternative for overcoming such limitations, Lee's L statistic for bivariate spatial association captures the spatial co-patterning by integrating a univariate spatial autocorrelation of each variable and their bivariate point-to-point associations [49]. Let $\widetilde{x_{i}}=\sum_{j} w_{i j} x_{j}$ and $\widetilde{y_{i}}=\sum_{j} w_{i j} y_{j}$ where $w_{i j}$ is an element of a row-standardized spatial weight matrix $\mathrm{W}$, and $r_{\widetilde{X}}, \widetilde{Y}$ be a Pearson's $\mathrm{R}$ between the spatial lag vectors of $X$ and $Y$. Then, $L$ statistics can be written as:

$$
L_{X, Y}=\sqrt{\frac{\sum_{i}\left(\widetilde{x}_{i}-\bar{x}\right)^{2}}{\sum_{i}\left(x_{i}-\bar{x}\right)^{2}}} \cdot r_{\tilde{X}, \widetilde{Y}}=\sqrt{\frac{\sum_{i}\left(\widetilde{x}_{i}-\bar{x}\right)^{2}}{\sum_{i}\left(x_{i}-\bar{x}\right)^{2}}} \cdot \sqrt{\frac{\sum_{i}\left(\widetilde{y_{i}}-\bar{y}\right)^{2}}{\sum_{i}\left(y_{i}-\bar{y}\right)^{2}}} \cdot \frac{\sum_{i}\left(\widetilde{x}_{i}-\overline{\tilde{x}}\right)\left(\widetilde{y_{i}}-\overline{\tilde{y}}\right)}{\sqrt{\sum_{i}\left(\widetilde{x}_{i}-\overline{\tilde{x}}\right)^{2}} \cdot \sqrt{\sum_{i}\left(\widetilde{y_{i}}-\overline{\tilde{y}}\right)^{2}}}
$$

As seen in the equation, the $L$ statistic is defined as an adjusted Pearson's $R$ between variables' spatial lags scaled by the square root of the bivariate spatial smoothing scalar. For environment and health data which tend to be spatially correlated, it can function as a superior alternative to Pearson's correlation coefficient, as it captures how much bivariate associations are spatially clustered. Likewise, the local measure of $L$ statistics $\left(L_{i}\right)$ can help investigate a bivariate spatial heterogeneity by detecting the local instability in relationships between the two variables $X$ and $Y$, which is presented as follows:

$$
L_{i}=\frac{n \cdot\left(\widetilde{x_{i}}-\bar{x}\right)\left(\widetilde{y_{i}}-\bar{y}\right)}{\sqrt{\sum_{i}\left(\widetilde{x}_{i}-\bar{x}\right)^{2}} \cdot \sqrt{\sum_{i}\left(\widetilde{y_{i}}-\bar{y}\right)^{2}}}
$$

In this study, both Lee's L and Pearson's R statistics were estimated and compared for each pair of air pollutants and allergic disease prevalence. The map of the local version of $L$ statistics for bivariate association of each pair was also created to demonstrate local patterns of spatial correlation between air pollutant concentration and the number of allergic disease patients, highlighting sub-regions into the four categories such as high-high and low-low locations (spatial clusters) and high-low and low-high locations (spatial outliers).

\section{Results}

A series of spatiotemporal maps for allergic disease patient counts in Seoul confirmed that our findings followed the national trend of decreasing prevalence of atopic dermatitis and asthma and the increasing prevalence of allergic rhinitis in South Korea [28,29]. However, as shown in Figure 2, the 4-year changes in spatiotemporal patterns for each allergic disease at the sub-district level in Seoul between 2011 and 2015 indicate that some sub-districts in Seoul do not follow the national patterns. Although the prevalence of allergic rhinitis and asthma has decreased in most of the sub-districts in Seoul since 2011, it remains similar or even increased in 10-20\% of the sub-districts. Only about $5 \%$ of the sub-districts were found to be regions where prevalence increased for all three diseases, which were dispersed throughout the city. Such spatial heterogeneity is even greater on allergic rhinitis. Roughly half of the sub-districts show decreasing patterns, while the other half display increasing patterns. Such differentials for trends of allergic disease prevalence highlight the limitations of previous study findings based on national aggregates because the spatially varied prevalence of allergic diseases at the finer levels of geography may have been masked by national level observation.

In addition, unless a significant and steady decrease in the associated risk factors was present, a decreasing prevalence trend could be a temporary phenomenon. Figure 3 shows a temporal pattern of yearly average concentrations for the four major ambient pollutants. All pollutants except carbon monoxide (CO) appear to be either steady or even increasing between 2011 and 2015 in Seoul. However, this kind of report could be misleading due to its aggregated process. This finding emphasizes the need for research efforts to explore the detailed spatial patterns of disease prevalence, as well as its underlying risk factors, in order to better interpret the temporal trends of allergic diseases. This study found substantial spatial heterogeneity in all four air pollutants, and that the patterns remained 
consistent over the five years. Maps of all four air pollutants by sub-district are available upon request from the authors.
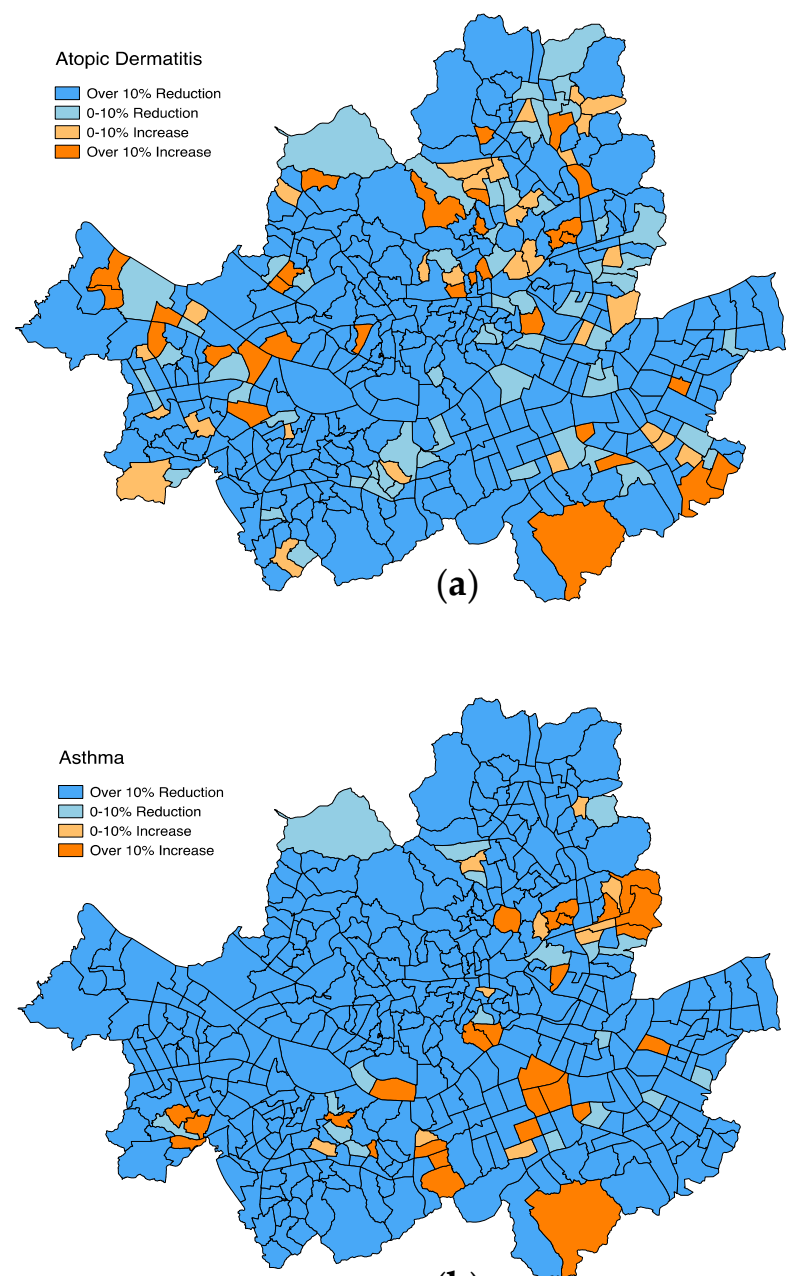

(b)

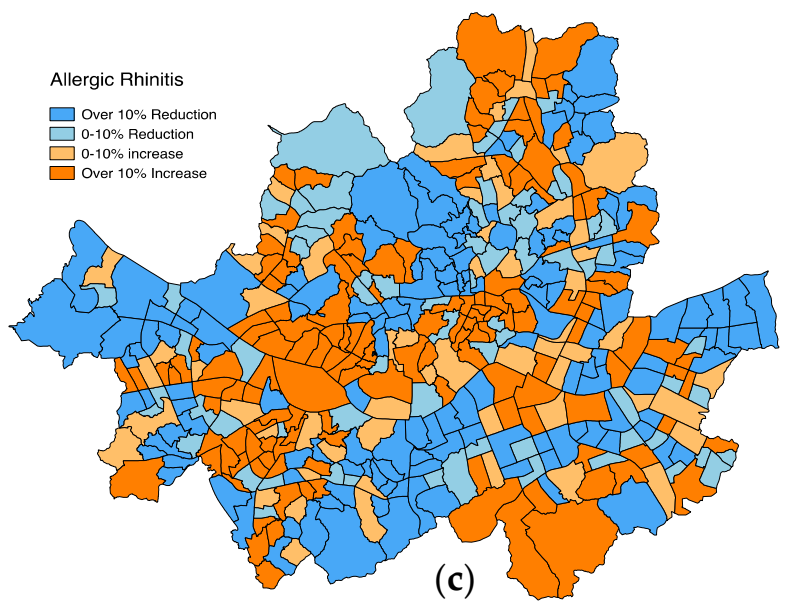

Figure 2. Changes in allergic disease prevalence at 423 sub-districts in Seoul (2011-2015): (a) atopic dermatitis; (b) asthma; (c) allergic rhinitis. 


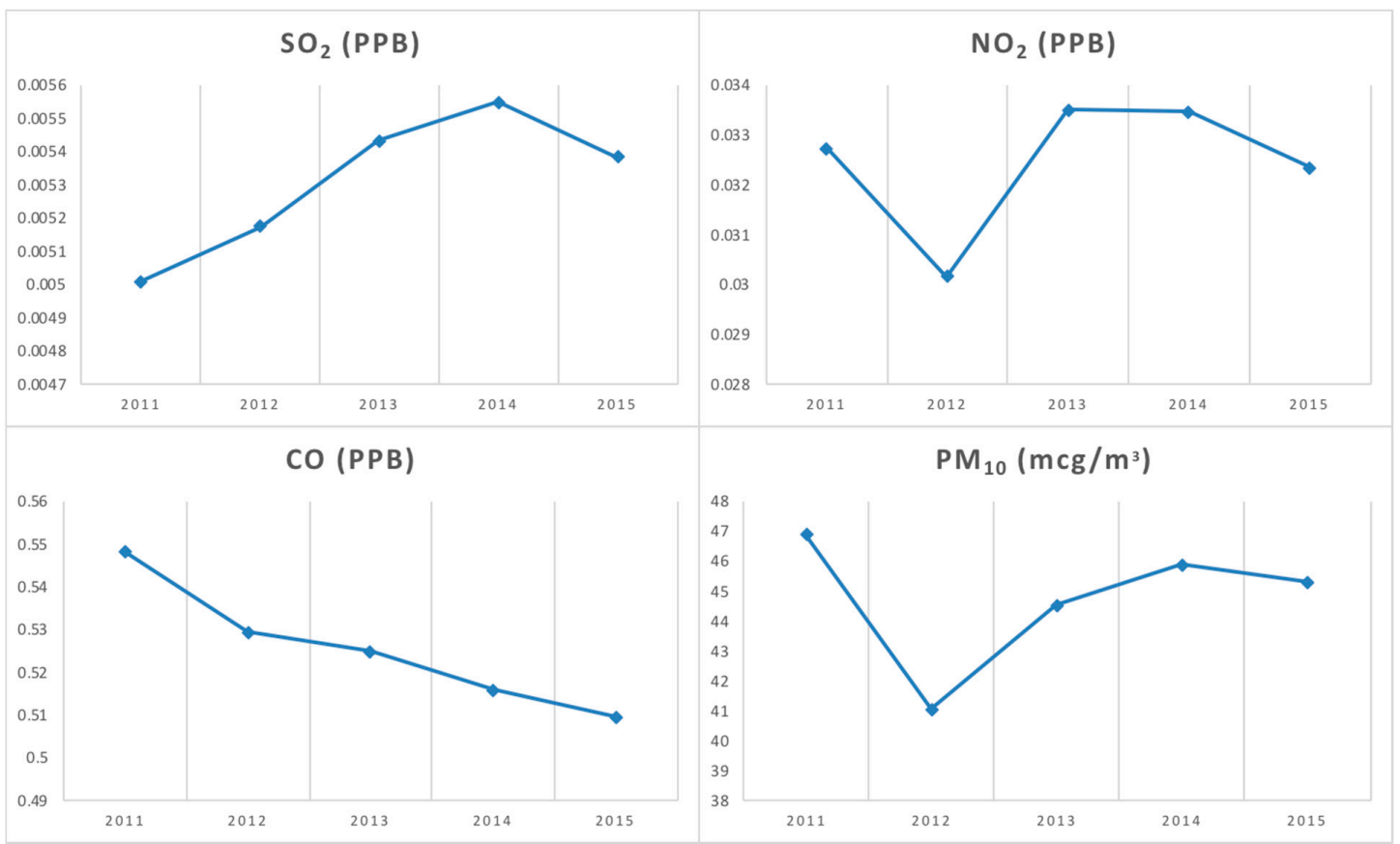

Figure 3. Changes in ambient air pollutants in Seoul between 2011 and 2015.

One of the intrinsic spatial aspects characterizing both ambient air pollutants and allergic diseases is spatial autocorrelation. Table 1 confirms a strong degree of spatial autocorrelation for each air pollutant and allergic disease, measured by a global Moran's coefficient ranging from $0.25-0.39$ for allergic diseases and 0.71-0.98 for air pollutants. Such a high level of spatial autocorrelation could make an aspatial bivariate association measure such as Pearson's correlation coefficient biased and inefficient, since it fails to address spatially-clustered phenomenon of the association.

Table 1. Univariate autocorrelation measures of allergic disease prevalence and air pollutants (global Moran's coefficient).

\begin{tabular}{ccccccc}
\hline & & $\mathbf{2 0 1 1}$ & $\mathbf{2 0 1 2}$ & $\mathbf{2 0 1 3}$ & $\mathbf{2 0 1 4}$ & $\mathbf{2 0 1 5}$ \\
\hline \multirow{3}{*}{ Allergic disease prevalence } & Allergic rhinitis (children under 12) & 0.260 & 0.295 & 0.291 & 0.284 & 0.290 \\
& Asthma (children under 12) & 0.290 & 0.339 & 0.296 & 0.359 & 0.346 \\
& Atopic dermatitis (children under 12) & 0.329 & 0.322 & 0.262 & 0.254 & 0.164 \\
\hline \multirow{4}{*}{ Ambient air pollutants } & $\mathrm{SO}_{2}$ & 0.844 & 0.957 & 0.984 & 0.982 & 0.872 \\
& $\mathrm{NO}_{2}$ & 0.833 & 0.711 & 0.761 & 0.876 & 0.901 \\
& $\mathrm{CO}$ & 0.796 & 0.891 & 0.618 & 0.957 & 0.969 \\
& $\mathrm{PM}_{10}$ & 0.961 & 0.971 & 0.982 & 0.838 & 0.946 \\
\hline
\end{tabular}

Note: All autocorrelation measures are significant at $p<0.01$.

Table 2 compares the Pearson's R and Lee's Global L measures for every pair between the four ambient air pollutants and the three allergic disease patient counts (only children under 12) during 2011 and 2015. The major findings from this table include: (1) for some pairs, the Lee's Global L is found to be significant with a correct sign (positive) while the Pearson's R is not significant; (2) for the pairs where the Pearson's R has a negative sign (which is counter-intuitive), the Lee's Global L becomes insignificant; (3) for the pairs where both measures are statistically significant at $5 \%$ level, the Lee's Global L tends to be smaller than the Pearson's R. Despite some fluctuation over time, this finding confirms the previous literature suggesting the significant bivariate association between atopic dermatitis and $\mathrm{PM}_{10}[17,50]$. Although the practical interpretation is limited due to the bivariate structure of the model, we argue that this evidence is sufficient to demonstrate that Lee's L measure could work as a superior alternative to Pearson's correlation coefficient, because the presence of spatial autocorrelation in the data may alter the parameter estimates and error probabilities of the metrics. 
Table 2. Bivariate correlation between air pollutants and allergic disease prevalence: Pearson's R vs. Lee's L.

\begin{tabular}{|c|c|c|c|c|c|c|c|c|c|c|c|}
\hline \multirow{2}{*}{ Air Pollutants } & \multirow{2}{*}{$\begin{array}{l}\text { Allergic Disease } \\
\text { Prevalence }\end{array}$} & \multicolumn{2}{|c|}{2011} & \multicolumn{2}{|c|}{2012} & \multicolumn{2}{|c|}{2013} & \multicolumn{2}{|c|}{2014} & \multicolumn{2}{|c|}{2015} \\
\hline & & Pearson's R & Lee's Global L & Pearson's R & Lee's Global L & Pearson's R & Lee's Global L & Pearson's R & Lee's Global L & Pearson's R & Lee's Global L \\
\hline \multirow{3}{*}{$\mathrm{SO}_{2}$} & Allergic rhinitis & NS & NS & NS & NS & NS & NS & -0.1 & NS & NS & NS \\
\hline & Asthma & NS & NS & NS & NS & NS & NS & NS & NS & NS & NS \\
\hline & Atopic dermatitis & NS & NS & NS & NS & NS & NS & -0.151 & NS & -0.114 & NS \\
\hline \multirow{3}{*}{$\mathrm{NO}_{2}$} & Allergic rhinitis & -0.113 & NS & -0.118 & NS & NS & NS & NS & NS & NS & NS \\
\hline & Asthma & NS & NS & -0.11 & NS & 0.119 & 0.091 & NS & NS & NS & 0.054 \\
\hline & Atopic dermatitis & NS & 0.063 & 0.132 & 0.112 & NS & 0.055 & 0.098 & 0.064 & NS & NS \\
\hline \multirow{3}{*}{$\mathrm{CO}$} & Allergic rhinitis & NS & NS & -0.099 & NS & NS & 0.039 & NS & NS & NS & NS \\
\hline & Asthma & NS & NS & NS & NS & NS & NS & -0.151 & NS & -0.11 & NS \\
\hline & Atopic dermatitis & 0.149 & 0.136 & 0.16 & 0.18 & NS & NS & NS & 0.059 & NS & 0.077 \\
\hline \multirow{3}{*}{$\mathrm{PM}_{10}$} & Allergic rhinitis & -0.114 & NS & NS & NS & NS & NS & -0.143 & NS & NS & NS \\
\hline & Asthma & -0.174 & NS & -0.114 & NS & NS & NS & -0.139 & NS & -0.127 & NS \\
\hline & Atopic dermatitis & 0.252 & 0.242 & NS & NS & NS & NS & NS & NS & NS & 0.045 \\
\hline
\end{tabular}

NS: Not significant at $p<0.05$. 
Figure 4 illustrates the case where the Lee's L becomes significant, while the Pearson's R shows no association. The classical scatterplot shows a one-to-one association between the crude measure of $\mathrm{PM}_{10}$ and atopic dermatitis patient counts (children under 12) over 423 sub-districts in Seoul during 2015. On the other hand, Lee's L scatterplot matches the Z-transformed spatial moving average for each of the same pair, which combine a univariate spatial autocorrelation of each variable with their bivariate point-to-point associations. The slope of the red line in the Lee's scatterplot indicates the $\mathrm{L}$ measure (0.045). Although it is small, the positive association between $\mathrm{PM}_{10}$ and atopic dermatitis could have been masked if examined by a classical bivariate association measure ignoring their spatial co-patterning.

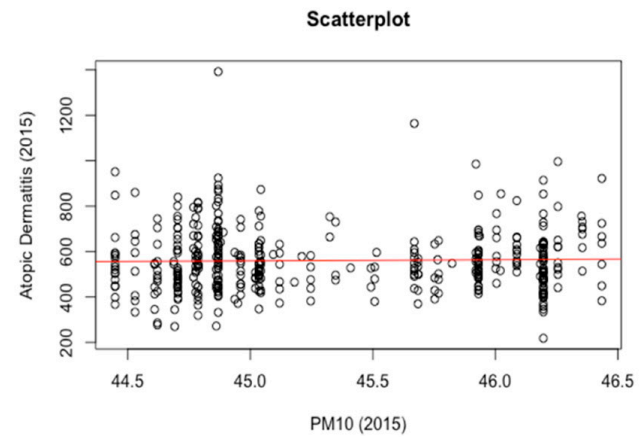

(a)

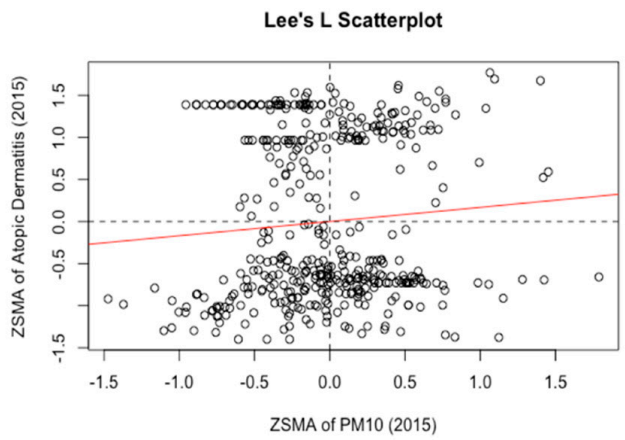

(b)

Figure 4. Scatterplots with (a) observed points and (b) Lee's L scatterplot with Z-transformed spatial moving average illustrating association between $\mathrm{PM}_{10}$ and atopic dermatitis (2015).

Despite some annual fluctuation and variation by age group, the Lee's L measures identify ozone as a major risk factor for asthma and allergic rhinitis, but $\mathrm{SO}_{2}, \mathrm{CO}$, and $\mathrm{PM}_{10}$ as core determinants for atopic dermatitis, during the past five years. However, such finding is also based on a global measure, and the local association patterns could vary by location. Similar to the Anselin's local indicators of spatial association (LISA), the Lee's L statistics can be calculated as a local-level indicator and illustrated as a map highlighting "hotspots" and "coldspots". "Hot spots" refer to a clustering of high air pollutant concentrations and clustering of high allergic disease prevalence, whereas "cold spots" refer to the clustering of low air pollutant concentrations and clustering of low allergic disease prevalence. Figure 5 demonstrates that there are several hot and cold spots for bivariate association between $\mathrm{PM}_{10}$ and atopic dermatitis (2015) in Seoul, along with other areas displaying negative associations between them (high-low and low-high). While much effort should be given to explaining the reason behind such local patterns, it would at least identify the areas where a specific air pollutant is considered to be contributing to a type of allergic disease, and thus, should be targeted for regional-specific intervention.



Figure 5. Map of Lee's Local L for bivariate association between $\mathrm{PM}_{10}$ and atopic dermatitis in Seoul. 


\section{Discussion}

The evidence from this study suggests that evaluating disease trends based on aggregated data may be misleading. Thus, it is crucial to include local-level spatiotemporal disease patterns to more effectively design environmental health policy. This argument is even more potent for allergic diseases, where various risk factors typically show substantial spatial and temporal variations. The driving factors for spatiotemporal variation in allergic diseases at different scales remain unknown and controversial, but this study highlights the role of air pollution in explaining the prevalence and severity of allergic diseases, and emphasizes the importance of taking spatial dynamics, particularly spatial autocorrelation, into careful consideration when measuring their ecological association. The findings in this study support previous ecological research by the authors that geographical variations in prevalence of allergic diseases may be explained by varying levels of air pollutants over space, rather than by individual risk factors [17]. This study also notes that recent decreasing trends of atopic dermatitis and asthma in South Korea reported in the literature may be artifacts of aggregation [28]. As such, they should be re-examined at a more granular level, particularly considering that most of the ambient pollutant concentrations have been either steady or even increasing recently in many areas.

Spatial data disaggregation is imperative to identifying regional-specific or area-specific risk factors, because it would be the first step to derive and establish a cost-effective targeted intervention in reducing the burden of allergic diseases. The comprehensive spatiotemporal patterns of allergic diseases at different scales in Korea presented in this paper could potentially be explained by further multivariate studies exploring the spatially-varying impacts of multiple indoor and outdoor risk factors if the data were available at more disaggregated spatial and temporal scales. Despite recent popularity in the use of Geographic Information Systems (GIS) in visualizing and analyzing spatial patterns for allergic diseases in South Korea [17], most data on disease prevalence, as well as environmental, meteorological, and socioeconomic risk factors, are still typically maintained at highly aggregated levels. For more widespread spatiotemporal environmental health research, the routine public health and environmental data in Korea, such as the National Health Insurance Corporation (NHIC) data and the NIER (National Institute of Environmental Research) air pollution data, need to be collected at more disaggregated administrative units, and be readily available to researchers and professionals in the field. Recent developments in big data analytics can greatly accelerate data-driven research and policy implementation using the large-sized spatiotemporal data on environmental risks and health outcomes [51].

However, there are several limitations inherent in this study. First of all, as this study focuses on the bivariate association between air pollution and allergic diseases, it rules out various environmental and socioeconomic variables or risk factors which may be related to at least one of the allergic diseases. As the level of various social and environmental risk factors tends to be spatially inconsistent and change over time, spatiotemporal variation in the prevalence of allergic diseases can be anticipated accordingly, although this paper looked into spatial and temporal heterogeneity separately, and ignored correlations among air pollutants. Their confounding effects may explain low correlation coefficients, measured by both Pearson's R and Lee's L statistics. As a matter of fact, the recent literature attempted to evaluate the impact of various allergens such as pollen count [52] and dust mites [53]. Studies on the effects of climate change on allergic diseases are still lacking in Korea, but the global rise in allergic disease prevalence may be directly or indirectly related to climate change and its aftermath [54,55]. Other limitations relate to the data source involved in this study. The patient count data measured by inpatient and outpatient medical treatment records may have overestimated or underestimated true disease burden due to error or bias in the data reporting process [29]. For instance, true level of exposure should be measured based on actual mobility patterns even beyond their administrative boundary, which cannot be captured by the aggregated patients count data. Moreover, the data fail to incorporate disease symptom severity, which tends to be closely related to air pollutant concentration [56]. Air pollutant concentration data-which are collected from only 25 monitoring stations in Seoul-had to be interpolated to the entire surface of Seoul to match with the patient 
count data collected at 423 sub-districts. The excessive level of spatial autocorrelation found in air pollutant concentrations could be due to this process of spatial estimation, and the results are subject to this type of spatial interpolation. Neither seasonality nor historical trends of both air pollutants and the disease outcomes were discussed in this study, as it focused more on spatial distribution of annually-aggregated data during the recent five years.

\section{Conclusions}

This study examines bivariate correlation between outdoor air pollutants and the prevalence of allergic diseases, highlighting the limitation of a non-spatial correlation measure, and suggesting an alternative to address spatial autocorrelation. It is also the first to compare two types of bivariate association measures—Lee's L statistics and Pearson's correlation coefficient—between ambient air pollution and allergic diseases in Korea at a highly disaggregated scale, emphasizing that not only spatially-clustered phenomenon of the association, but also local instability in their relationships, should be considered in investigating the environmental impact on diseases. Although the association between atopic dermatitis and $\mathrm{PM}_{10}$ is still under debate, the findings presented in this study underscore the role of spatial analysis in investigating the contribution of local-level spatiotemporal dynamics of air pollution to trends and distribution of allergic diseases.

Author Contributions: Conceptualization, D.K. and S.S.; Data curation, S.M.; Formal analysis, D.K.; Funding acquisition, S.S.; Methodology, D.K.; Project administration, S.S.; Supervision, S.S.; Visualization, D.K. and S.M.; Writing-original draft, D.K. and S.M.; Writing-review \& editing, D.K., S.S., Z.S., S.K. and M.K.

Funding: This research was funded by Ministry of Environment, Republic of Korea (grant number 2017001350002).

Acknowledgments: This study was supported by a fund (“Environmental Technology Development Project: Development of Receptor-centered Exposure Assessment Methodology and Service platform, project No. 2017001350002) by Ministry of Environment, Republic of Korea. This support is greatly appreciated. All authors declare they have no actual or potential competing financial interest.

Conflicts of Interest: The authors declare no conflict of interest.

\section{References}

1. Institute of Medicine. Clearing the Air: Asthma and Indoor Air Exposures; The National Academies Press: Washington, DC, USA, 2000.

2. Borish, L. Genetics of allergy and asthma. Ann. Allergy Asthma Immunol. 1999, 82, 413-426. [CrossRef]

3. Prescott, S.; Tang, M. The australasian society of clinical immunology and allergy position statement: Summary of allergy prevention in children. Med. J. Aust. 2005, 182, 464-467. [PubMed]

4. Bernard, S.M.; Samet, J.M.; Grambsch, A.; Ebi, K.L.; Romieu, I. The potential impacts of climate variability and change on air pollution-related health effects in the United States. Environ. Health Perspect. 2001, 109, 199-209. [PubMed]

5. Wong, T.W.; Tam, W.; Yu, I.T.S.; Wun, Y.T.; Wong, A.H.; Wong, C.M. Association between air pollution and general practitioner visits for respiratory diseases in Hong Kong. Thorax 2006, 61, 585-591. [CrossRef] [PubMed]

6. Piantadosi, S.; Byar, D.P.; Green, S.B. The ecological fallacy. Am. J. Epidemiol. 1988, 127, 893-904. [CrossRef] [PubMed]

7. Openshaw, S. The Modifiable Areal Unit Problem; Geobooks: Norwich, UK, 1983.

8. Bruno, F.; Cameletti, M.; Franco-Villoria, M.; Greco, F.; Ignaccolo, R.; Ippoliti, L.; Valentini, P.; Ventrucci, M. A survey on ecological regression for health hazard associated with air pollution. Spat. Stat. 2016, 18, 276-299. [CrossRef]

9. Akinbami, L.J.; Schoendorf, K.C. Trends in childhood asthma: Prevalence, health care utilization, and mortality. Pediatrics 2002, 110, 315-322. [CrossRef] [PubMed]

10. Schuurman, N.; Bell, N.; Dunn, J.R.; Oliver, L. Deprivation indices, population health and geography: An evaluation of the spatial effectiveness of indices at multiple scales. J. Urban Health 2007, 84, 591-603. [CrossRef] [PubMed] 
11. Dark, S.J.; Bram, D. The modifiable areal unit problem (MAUP) in physical geography. Prog. Phys. Geogr. 2007, 31, 471-479.

12. Gee, G.C.; Payne-Sturges, D.C. Environmental health disparities: A framework integrating psychosocial and environmental concepts. Environ. Health Perspect. 2004, 112, 1645-1653. [CrossRef] [PubMed]

13. Hales, S.; Weinstein, P.; Souares, Y.; Woodward, A. El niño and the dynamics of vectorborne disease transmission. Environ. Health Perspect. 1999, 107, 99-102. [PubMed]

14. Cocco, P.; Kazerouni, N.; Zahm, S.H. Cancer mortality and environmental exposure to dde in the United States. Environ. Health Perspect. 2000, 108, 1-4. [CrossRef] [PubMed]

15. Graber, L.K.; Asher, D.; Anandaraja, N.; Bopp, R.F.; Merrill, K.; Cullen, M.R.; Luboga, S.; Trasande, L. Childhood lead exposure after the phaseout of leaded gasoline: An ecological study of school-age children in Kampala, Uganda. Environ. Health Perspect. 2010, 118, 884-889. [CrossRef] [PubMed]

16. Ko, F.; Tam, W.; Wong, T.; Lai, C.; Wong, G.; Leung, T.F.; Ng, S.; Hui, D. Effects of air pollution on asthma hospitalization rates in different age groups in Hong Kong. Clin. Exp. Allergy 2007, 37, 1312-1319. [CrossRef] [PubMed]

17. Seo, S.; Kim, D.; Min, S.; Paul, C.; Yoo, Y.; Choung, J.T. Gis-based association between pm10 and allergic diseases in seoul: Implications for health and environmental policy. Allergy Asthma Immunol. Res. 2016, 8, 32-40. [CrossRef] [PubMed]

18. Holguin, F. Traffic, outdoor air pollution, and asthma. Immunol. Allergy Clin. N. Am. 2008, 28, 577-588. [CrossRef] [PubMed]

19. Wong, G.; Ko, F.; Lau, T.; Li, S.; Hui, D.; Pang, S.; Leung, R.; Fok, T.; Lai, C. Temporal relationship between air pollution and hospital admissions for asthmatic children in Hong Kong. Clin. Exp. Allergy 2001, 31, 565-569. [CrossRef] [PubMed]

20. Walters, S.; Griffiths, R.; Ayres, J. Temporal association between hospital admissions for asthma in birmingham and ambient levels of sulphur dioxide and smoke. Thorax 1994, 49, 133-140. [CrossRef] [PubMed]

21. Kimes, D.; Levine, E.; Timmins, S.; Weiss, S.R.; Bollinger, M.E.; Blaisdell, C. Temporal dynamics of emergency department and hospital admissions of pediatric asthmatics. Environ. Res. 2004, 94, 7-17. [CrossRef]

22. Griffith, D.A. Spatial Autocorrelation: A Primer; Association of American Geographers: Washington, DC, USA, 1987.

23. Haining, R. Bivariate correlation with spatial data. Geogr. Anal. 1991, 23, 210-227. [CrossRef]

24. Park, Y.M.; Kim, Y. A spatially filtered multilevel model to account for spatial dependency: Application to self-rated health status in South Korea. Int. J. Health Geogr. 2014, 13, 6. [CrossRef] [PubMed]

25. Legendre, P. Spatial autocorrelation: Trouble or new paradigm? Ecology 1993, 74, 1659-1673. [CrossRef]

26. Shankardass, K.; Jerrett, M.; Dell, S.; Foty, R.; Stieb, D. Spatial analysis of exposure to traffic-related air pollution at birth and childhood atopic asthma in Toronto, Ontario. Health Place 2015, 34, 287-295. [CrossRef] [PubMed]

27. Crighton, E.J.; Feng, J.; Gershon, A.; Guan, J.; To, T. A spatial analysis of asthma prevalence in Ontario. Can. J. Public Health 2012, 103, 384-389.

28. Kim, B.-K.; Kim, J.-Y.; Kang, M.-K.; Yang, M.-S.; Park, H.-W.; Min, K.-U.; Cho, S.-H.; Kang, H.-R. Allergies are still on the rise? A 6-year nationwide population-based study in Korea. Allergol. Int. 2016, 65, 186-191. [CrossRef] [PubMed]

29. Lee, J.; Yang, H.; Kim, M.; Kim, J.; Ahn, K. Is the prevalence of atopic dermatitis in korean children decreasing?: Analysis of the national statistics data, 2009-2014. Asian Pac. J. Allergy Immunol. 2017, 35, 144-149. [PubMed]

30. Ghim, Y.S.; Moon, K.-C.; Lee, S.; Kim, Y.P. Visibility trends in Korea during the past two decades. J. Air Waste Manag. Assoc. 2005, 55, 73-82. [CrossRef] [PubMed]

31. National Institute of Environmental Research. Annual Report of Air Quality in Korea 2015. Available online: http:/ / webbook.me.go.kr/DLi-File/NIER/09/022/5618423.pdf (accessed on 13 September 2017).

32. Leem, J.-H.; Kaplan, B.M.; Shim, Y.K.; Pohl, H.R.; Gotway, C.A.; Bullard, S.M.; Rogers, J.F.; Smith, M.M.; Tylenda, C.A. Exposures to air pollutants during pregnancy and preterm delivery. Environ. Health Perspect. 2006, 114, 905-910. [CrossRef] [PubMed]

33. Ministry of Environment. What Is Fine Dust? Available online: http://m.me.go.kr/issue/finedust/ebook. pdf (accessed on 13 September 2017). 
34. Son, J.-Y.; Bell, M.L.; Lee, J.-T. Individual exposure to air pollution and lung function in Korea: Spatial analysis using multiple exposure approaches. Environ. Res. 2010, 110, 739-749. [CrossRef] [PubMed]

35. Kim, B.-J.; Hong, S.-J. Ambient air pollution and allergic diseases in children. Korean J. Pediatr. 2012, 55, 185-192. [CrossRef] [PubMed]

36. Oh, W.S.; Hieu, N.C.; Kim, S.M.; Sohn, J.W.; Heo, J. Spatial autocorrelation of disease prevalence in south korea using 2012 community health survey data. J. Korean Soc. Surv. Geodesy Photogramm. Cartogr. 2016, 34, 253-262. [CrossRef]

37. Hasegawa, K.; Tsugawa, Y.; Tsai, C.L.; Brown, D.F.; Camargo, C.A. Frequent utilization of the emergency department for acute exacerbation of chronic obstructive pulmonary disease. Respir. Res. 2014, 15, 1-13. [CrossRef] [PubMed]

38. Steer, J.; Gibson, G.J.; Bourke, S.C. Predicting outcomes following hospitalization for acute exacerbations of copd. QJM Int. J. Med. 2010, 103, 817-829. [CrossRef] [PubMed]

39. Zheng, X.; Ding, H.; Jiang, L.; Chen, S.; Zheng, J.; Qiu, M.; Zhou, Y.; Chen, Q.; Guan, W. Association between air pollutants and asthma emergency room visits and hospital admissions in time series studies: A systematic review and meta-analysis. PLoS ONE 2015, 10, e0138146. [CrossRef] [PubMed]

40. Romangnani, S. The increased prevalence of allergy and the hygiene hypothesis: Missing immune deviation, reduced immune suppression, or both? Immunology 2004, 112, 352-363. [CrossRef] [PubMed]

41. Spergel, J.M.; Paller, A.S. Atopic dermatitis and the atopic march. J. Allergy Clin. Immunol. 2003, 112, S118-S127. [CrossRef] [PubMed]

42. Kim, Y.; Kim, J.; Han, Y.; Lee, B.; Choi, D.; Cheong, H.; Jeon, B.; Oh, I.; Bae, G.; Lee, J.; et al. Comparison of diverse estimation methods for personal exposure to air pollutants and associations with allergic symptoms: The allergy \& gene-environment link (angel) study. Sci. Total Environ. 2017, 579, 1127-1136. [PubMed]

43. Joseph, J.; Sharif, H.O.; Sunil, T.; Alamgir, H. Application of validation data for assessing spatial interpolation method for 8-h ozone or other sparsely monitored constituents. Environ. Pollut. 2013, 178, 411-418. [CrossRef] [PubMed]

44. Liao, D.; Peuquet, D.J.; Duan, Y.; Whitsel, E.A.; Dou, J.; Smith, R.L. GIS approaches for the estimation of residential-level ambient PM concentrations. Environ. Health Perspect. 2006, 114, 1374-1380. [CrossRef] [PubMed]

45. Dormann, C.; McPherson, J.M.; Araujo, M.B.; Bivand, R.; Bolliger, J.; Carl, G.; Davies, R.G.; Hirzel, A.; Jetz, W.; Kissling, W.D.; et al. Methods to account for spatial autocorrelation in the analysis of species distributional data: A review. Ecography 2007, 30, 609-628. [CrossRef]

46. Hu, Z.; Rao, K. Particulate air pollution and chronic ischemic heart disease in the eastern United States: A county level ecological study using satellite aerosol data. Environ. Health 2009, 8, 1-10. [CrossRef] [PubMed]

47. Hendryx, M.; Fedorko, E.; Anesetti-Rothermel, A. A geographical information system-based analysis of cancer mortality and population exposure to coal mining activities in West Virginia, United States of America. Geospat. Health 2010, 4, 243-256. [CrossRef] [PubMed]

48. Anselin, L.; Syabri, I.; Kho, Y. Geoda: An introduction to spatial data analysis. Geogr. Anal. 2006, 38, 5-22. [CrossRef]

49. Lee, S. Developing a bivariate spatial association measure: An integration of Pearson's R and Moran's I. J. Geogr. Syst. 2001, 3, 369-385. [CrossRef]

50. Ngoc, L.; Park, D.; Lee, Y.; Lee, Y. Systematic review and meta-analysis of human skin diseases due to particulate matter. Int. J. Environ. Res. Public Health 2017, 14, 1458. [CrossRef] [PubMed]

51. Ram, S.; Zhang, W.; Williams, M.; Pengetnze, Y. Predicting asthma-related emergency department visits using big data. IEEE J. Biomed. Health Inform. 2015, 19, 1216-1223. [CrossRef] [PubMed]

52. Oh, Y.; Kim, H.; Kang, I.; Cheong, J.; Kim, S.; Kook, M.; Kim, B.; Lee, H.; Oh, J. Evaluation of the relationship between pollen count and the outbreak of allergic diseases. Pediatr. Allergy Respir. Dis. 2009, 19, 354-364.

53. Kim, K.; Kim, K.; Lee, S.; Park, H.; Lee, Y.; Nahm, D.; Son, C.; Yang, D.; Roh, M.; Choi, P.; et al. Sensitization rates for inhalant allergens in patients with respiratory allergy in Busan. Korean J. Asthma Allergy Clin. Immunol. 2005, 25, 59-63.

54. Shea, K.; Truckner, R.; Weber, R.; Peden, D. Climate change and allergic disease. J. Allergy Clin. Immunol. 2008, 122, 443-453. [CrossRef] [PubMed] 
55. Barnes, C.; Alexis, N.; Bernstein, J.; Cohn, J.; Demain, J.; Horner, E.; Levetin, E.; Nei, A.; Phipatanakul, W. Climate change and our environment: The effect on respiratory and allergic disease. J. Allergy Clin. Immunol. Pract. 2013, 1, 137-141. [CrossRef] [PubMed]

56. Slaughter, J.C.; Lumley, T.; Sheppard, L.; Koenig, J.; Shapiro, G. Effects of ambient air pollution on symptom severity and medication use in children with asthma. Ann. Allergy Asthma Immunol. 2003, 91, 346-353. [CrossRef]

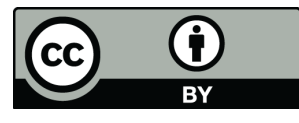

(C) 2018 by the authors. Licensee MDPI, Basel, Switzerland. This article is an open access article distributed under the terms and conditions of the Creative Commons Attribution (CC BY) license (http:/ / creativecommons.org/licenses/by/4.0/). 